\title{
Focus on mechanical assist devices for the treatment of heart failure
}

\author{
Philip A Poole-Wilson
}

Severe heart failure (HF) is becoming more prevalent as the population ages and a greater number of patients survive initial damage to the heart. In the last few decades, age-adjusted life expectancy has been prolonged and patient quality of life improved by judicious treatment with diuretics, angiotensin-converting-enzyme inhibitors, angiotensin-receptor blockers, and $\beta$-blockers. Cardiac transplantation was the traditional option for the inevitable worsening of $\mathrm{HF}$, but the number of procedures carried out is falling for many reasons and transplantation is an unrealistic solution for elderly patients. The alternative strategies are cell growth, regeneration, or replacement-approaches that are in an early stage of development-or the use of mechanical assist devices to support the function of the heart. There are, however, three major problems with the use of such devices, which are highlighted in the Viewpoint articles in this issue of Nature Clinical Practice Cardiovascular Medicine.

The first difficulty is that the benefit, if any, of mechanical assist device therapy varies with diagnosis. For example, cardiogenic shock is a different clinical entity to deterioration of chronic HF, where destination therapy is now a practical consideration. The first person in the world ever to have a left ventricular assist device implanted, with the clinical intention of it being a lifetime treatment, lived for 7.5 years. An essential requirement in assist device research is that the clinical diagnosis is accurate and made before implantation. Physicians should declare explicitly whether the device is intended for transient support, bridge to recovery, bridge to transplantation, or destination therapy.

The second major problem arises from advances in medical care. A recent report indicates that life expectancy among patients
There is

currently an

overwhelming

need for

formal trials

evaluating

mechanical

assist device

therapy in

patients

with various

diagnoses...

PA Poole-Wilson is an Advisory Board member of Nature Clinical Practice Cardiovascular Medicine.

\section{Competing interests}

The author declared no competing interests.

www.nature.com/clinicalpractice doi:10.1038/ncpcardio1114 in the US listed as status 2 for transplantation is similar whether or not they receive a donor (Lietz K and Miller LW [2007] J Am Coll Cardiol 50: 1282-1290). This finding raises the question of whether transplantation should be subject to a clinical trial. A review from Germany suggested that only those with the very severest degrees of HF benefit from heart transplantation, and then only for a short period of time (Deng MC et al. [2000] BMJ 321: 540545). Furthermore, there is concern about the ability of medical personnel to estimate prognosis in individual patients. The situation has been made more complex by the availability of other means of cardiac support, such as implantable cardioverter-defibrillators and cardiac resynchronization therapy.

The final issue is how the efficacy and safety of mechanical assist devices should be determined in selected patients. To date, only one randomized trial has been undertaken (Rose EA et al. [2001] N Engl J Med 345: 1435-1443) and there is a need for rigorous clinical trials to evaluate the benefit of mechanical assist devices in carefully selected and documented groups of patients. Patients should not be misinformed that they are receiving the latest effective therapy, when the only evidence for this approach is observational data or clinical opinion. The idea that a patient is advised to undergo device implantation to establish safety, or so that the medical profession can learn how to undertake the procedure, is risible.

There is currently an overwhelming need for formal trials evaluating mechanical assist device therapy in patients with various diagnoses, in order to determine safety and efficacy, to better inform patients of their clinical options, and to direct expensive medical care in an appropriate direction. 